\title{
Performance of OFDM in Radio Mobile Channel
}

\author{
Mohamed Tayebi and Mrahi Bouziani \\ Tayebi_med@hotmail.com
}

\begin{abstract}
The OFDM has emerged in the second half of last century. However, it remains a technique used in broadband wireless communication systems. Which limits its use is its vulnerability to frequency shifts. The Doppler effect and imperfections of the local oscillators of the transmitter and receiver are at the origin of these shifts. The imperfections of local oscillators shift the spectrum of the OFDM signal, while the Doppler-effect expands it (or compresses it). In this paper, the impact of the Doppler-effect on the OFDM modulation is analyzed, we give a new expression of the contribution of each sub-carrier at the transmitter on each subcarrier demodulated at the receiver. Using the method of inter-carrier interference self-cancellation proposed by Zhao and Haggman, the system becomes more efficient.
\end{abstract}

Keywords: Orthogonal frequency division multiplexing (OFDM), carrier frequency offset (CFO), Doppler effect, Inter carriers Interferences (ICI), carrier to interferences ratio (CIR).

\section{Introduction}

The high spectral efficiency and robustness against multipath make OFDM one of the most promising techniques in wireless communications systems. Currently, OFDM has been adapted to the digital audio and video broadcasting (DAB/DVB) system, highspeed wireless local area networks (WLAN) such as IEEE802.11, HIPERLAN II, ADSL, and recently in the optical communication [1]. OFDM divides the available spectrum into $\mathrm{N}$ equal intervals, the symbols are sent in parallel channels with lower flow rates [2].The high mobility is a major challenge for wireless communications. It creates frequency shifts in part caused by the Doppler effect [3].These offsets destroy the orthogonality between subcarriers of the OFDM signal, and generates inter-carrier interference responsible for the degradation of system performance [4]. A number of studies has been developed to eliminate inter-carrier interference [5],[6], we will be interested by the method of Inter-carrier interferences self-cancellation for the first time proposed by Zhao and Haggman [6]. In this paper we study the superposition of the offset caused by the imperfections of local oscillators of the transmitter and receiver, and the Doppler-effect and its impact on all the subcarriers that constitute the OFDM signal. To improve system performance, we used the method of ICI self-interference [6]. This work was structured as follows, section 2 describes the nature of the frequency shift. The third topic focuses on the nature of the interference generated. The fourth section 
gives the system performance in terms of CIR and we apply an existing method for eliminating interference[6]; and last we finalize with a conclusion.

\section{Frequency Shifts in a Mobile Radio Channel}

In this section we will introduce the impact of the Doppler-effect on all the subcarriers that constitute the OFDM signal. We will assume that the local oscillators of the transmitter and receiver do not wobble at the same frequency. At the transmitter, the frequencies are equal to:

$$
f_{k}=\frac{k}{T} \quad k=0,1, \ldots, N-1
$$

Where $k$ represent the index of subcarriers. Then at the receiver, frequencies are equal to:

$$
f_{k}=\frac{k}{T}+\Delta f \quad k=0,1, \ldots, N-1
$$

Where $\mathrm{N}$ is the total number of subcarriers and $\Delta \mathrm{f}$ the frequency difference between transmitter and receiver. More if the receiver moves to the transmitter at a relative speed $\mathrm{v}$, the Doppler-effect is manifested and the frequency received signal will be equal to:

$$
f_{k}=\left(\frac{k}{T}+\Delta f\right)\left(1 \pm \frac{v}{c} \cos \alpha\right)
$$

Where $c$ is the speed of light, and $\alpha$ the angle between the velocity and direction of the electromagnetic wave. Then $f_{k}$ can be written:

$$
f_{k}=\frac{1}{T}(k+\varepsilon)(1+\beta)
$$

With

$$
\beta= \pm \frac{v}{c} \cos \alpha
$$

Where $\varepsilon$ is the normalized frequency offset due to the imperfections of local oscillators. The total normalized offset $\delta$ will be equal to:

$$
\delta(k)=\beta k+\varepsilon(1+\beta)
$$

$\delta$ is proportional to the index $k$ of the subcarrier. In other words, the offset is different for each subcarrier, we are thus faced with a compression of the spectrum of OFDM signal if $\beta$ is negative, or otherwise, with an expansion of the spectrum in if $\beta$ is positive. For a given connection, the imperfections of the oscillators generate a constant normalized offset $\varepsilon$, while the Doppler effect produces a shift proportional to the index of the subcarrier $\mathrm{k}$. 


\section{Inter-carrier Interferences}

In the time domain, the OFDM signal is written as:

$$
x(t)=\sum_{k=0}^{N-1} X(k) \exp \left(j 2 \pi f_{k} t\right)
$$

Where $X(k)$ is the signal to be transmitted and $f_{k}$ represents the $\mathrm{N}$ different frequencies of sub-carriers that form the OFDM signal. By discretizing the signal $x(t)$, the signal obtained at the receiver is then:

$$
\begin{gathered}
y(n)=\sum_{k=0}^{N-1} X(k) \exp \left(j 2 \pi \frac{n}{N}(k+\varepsilon)(1+\beta)\right) \\
=\exp \left(j 2 \pi \frac{n \varepsilon}{N}(1+\beta)\right) \times \sum_{k=0}^{N-1} X(k) \exp \left(\left(j 2 \pi \frac{n k}{N}(1+\beta)\right)\right.
\end{gathered}
$$

The signal $y(n)$ is out of phase with the original signal, this is due to imperfections of local oscillators. The signal undergoes an expansion (or compression) of the signal, since the Doppler-effect affects each subcarrier differently. At the end of the FFT, we get the symbols $Y(k)$, their expression is given by the equation:

$$
Y(k)=\frac{1}{N} \sum_{n=0}^{N-1} \sum_{k=0}^{N-1} X(k) \exp \left(\left(j 2 \pi \frac{n}{N}(k-m+k \beta+\varepsilon(1+\beta))\right)\right.
$$

The complex coefficients will be expressed as:

$$
\begin{aligned}
S_{k m}(k-m)= & \frac{\sin \pi(k-m+k \beta+\varepsilon(1+\beta))}{N \sin \frac{\pi}{N}(k-m+k \beta+\varepsilon(1+\beta))} \\
& \quad \times \exp j \pi\left(1-\frac{1}{N}\right)(k-m+k \beta+\varepsilon(1+\beta))
\end{aligned}
$$

The amplitudes of the complex coefficients have different values for positive and negative relative velocities. The influence of the subcarrier $\mathrm{m}$ on subcarrier $\mathrm{k}$ is different. Figure 1 shows the variation of the amplitudes of complex coefficients for a fixed value of the normalized frequency offset $\varepsilon$, but with three values of relative speed $\beta$, the first zero, the second positive and the last negative. 


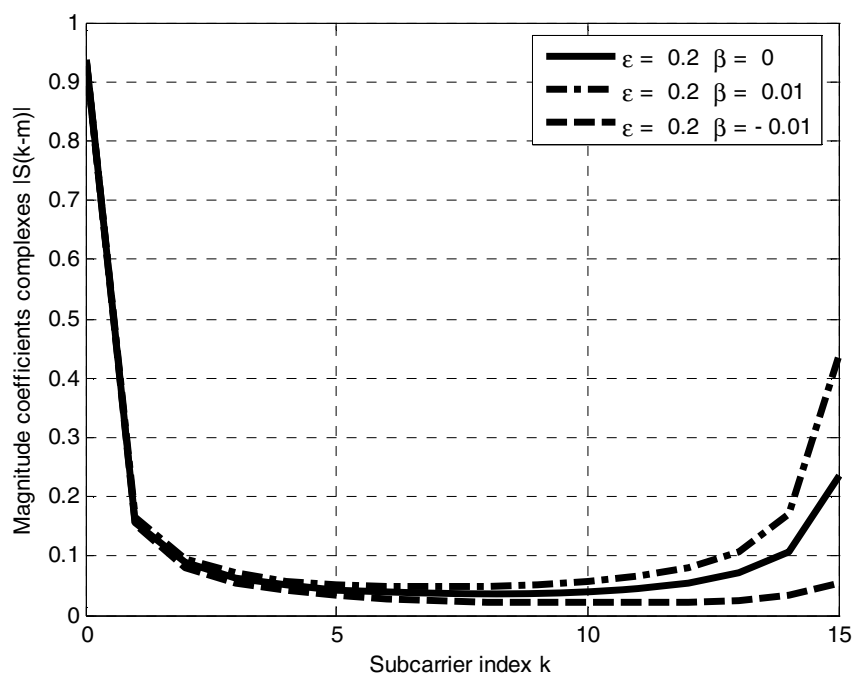

Fig. 1. Plot of the amplitudes of complex coefficients as a function of the index $k$ of subcarriers for different values of relative speed

\section{System Performances}

The useful signal, is assigned the complex coefficient $S_{k k}(0)$ calculated for the indices $k=m$ :

$$
S_{k k}(0)=\frac{\sin \pi(k \beta+\varepsilon(1+\beta))}{N \sin \frac{\pi}{N}(k \beta+\varepsilon(1+\beta))} \exp j \pi\left(1-\frac{1}{N}\right)(k \beta+\varepsilon(1+\beta))
$$

The complex coefficients of the different sub-carriers are not the same magnitude, since they depend on the index of the subcarrier $k$.. Figure 2 shows the degradation of the useful signal as a function of the index $k$. Similarly, we will study all the interference caused by the subcarriers index $k \neq m$.. The power of interference $\sigma_{I C I}$ is given by the relation:

$$
\sigma_{I C I}=\sum_{\substack{k=2 \\ k \neq m}}^{N-1}\left|S_{k m}(k-m)\right|^{2}
$$




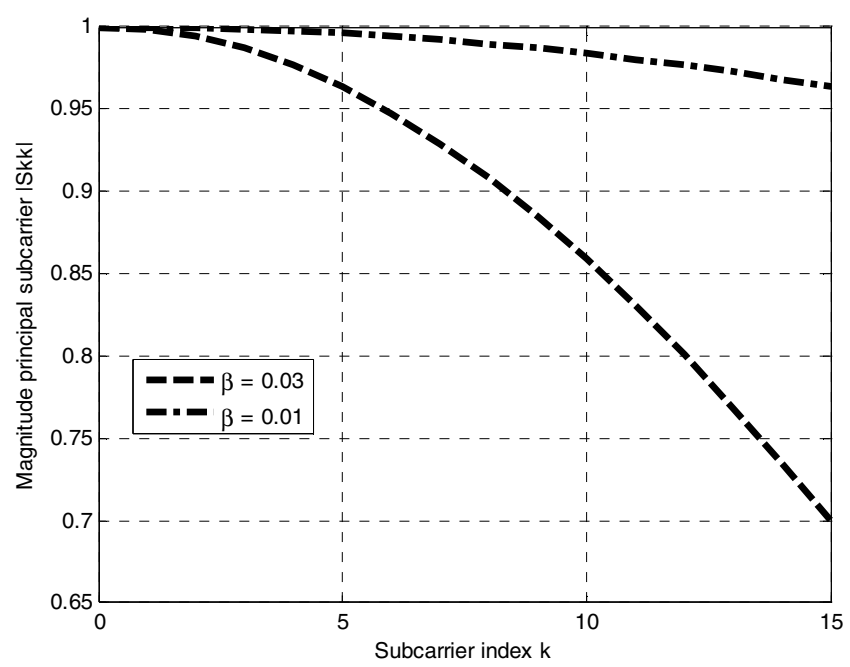

Fig. 2. Amplitude of the subcarrier based on the main index subcarriers $k$

This power is calculated for different values of $\mathrm{m}$. To study the system performance, we calculated the ratio of carrier power on the power of all interference, it is noted by CIR. In the case studied, the frequency shift is proportional to the index of the subcarrier, which implies that the CIR is different for different subcarriers. Its expression is given by the relation:

$$
C I R_{k m}(\varepsilon, \beta)=\frac{\left|S_{k m}(0)\right|^{2}}{\sum_{\substack{k=2 \\ k \neq m}}^{N-1}\left|S_{k m}(k-m)\right|^{2}}
$$

Figure 3 shows that for two different sub-carriers, the difference can reach $10 \mathrm{~dB}$. To improve system performance, we use the method of ICI self-cancellation. The parallel data streams are remapped as the form of :

$$
X(1)=-X(0), X(3)=-X(2), \ldots, X(N-1)=-X(N-2)
$$

The CIR is given by the relation:

$$
\begin{aligned}
& \operatorname{CIR}_{k m}(\varepsilon, \beta) \\
& =\frac{\left|2 * S_{k k}(0)-S_{k k}(1)-S_{k k}(-1)\right|^{2}}{\sum_{\substack{k=2 \\
k \neq m \\
k \text { even }}}^{N-1}\left|2 * S_{k m}(k-m)-S_{k m}(k-m+1)-S_{k m}(k-m-1)\right|^{2}}
\end{aligned}
$$




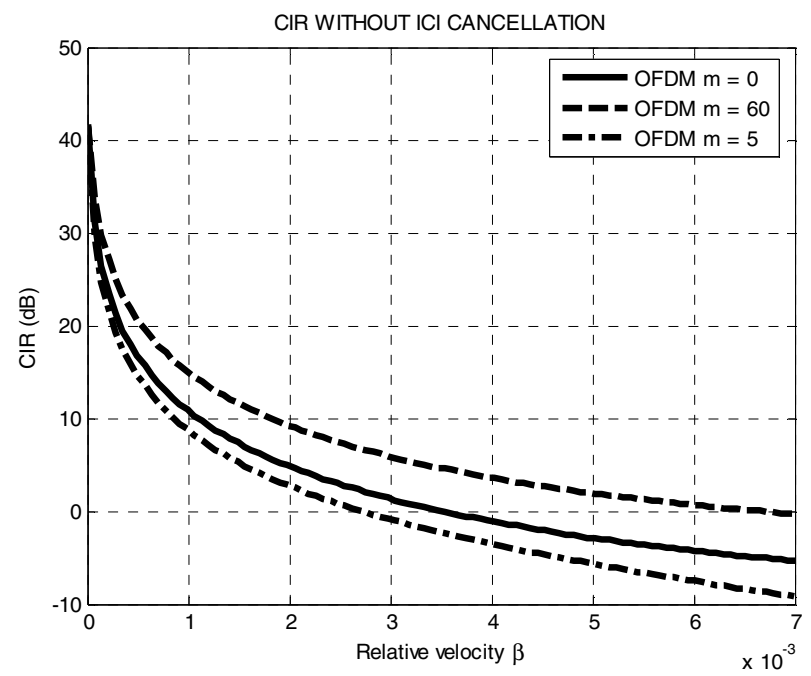

Fig. 3. Plot of the CIR as a function of relative velocity for different values of $m$

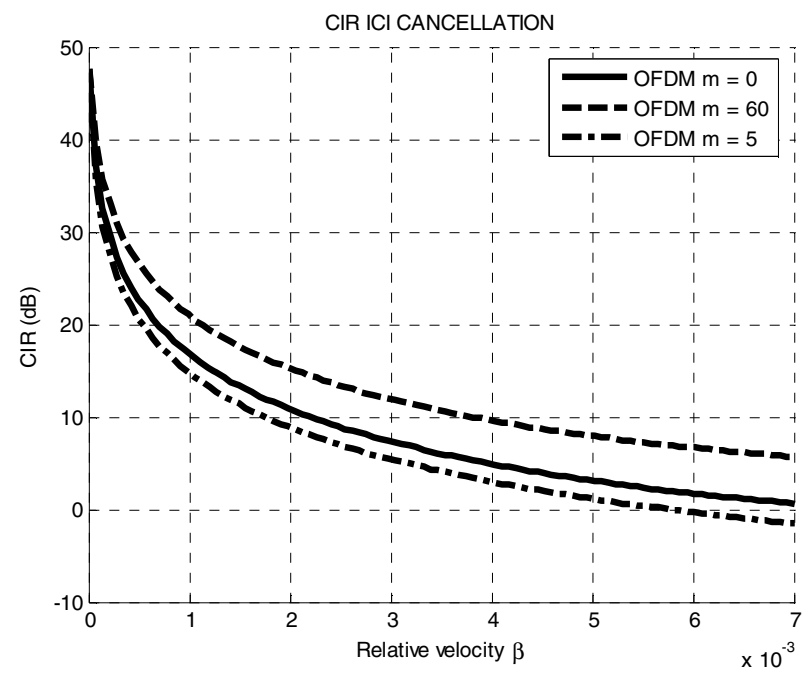

Fig. 4. Plot of CIR with ICI cancellation as a function of the speed for different values of $m$

Figure 3 plots the performance without using the method of ICI self- cancellation. However, Figure 4 plots the performance using the cited method. The gain varies from 7 to $10 \mathrm{~dB}$ for different subcarriers. 


\section{Conclusion}

In this paper, we analyzed the performance of OFDM systems in a mobile radio channel. This analysis enabled us to lead to new mathematical expressions. Indeed, the Doppler effect present in the mobile radio channels, shifts not only the carrier frequency, but extended (or compress) the spectrum of OFDM signal because it shifts each subcarrier differently. So we calculated the performance for each subcarrier. Applying the method of ICI cancellation, we improved the system performance. This improvement has resulted in a gain ranging from 7 to $10 \mathrm{Db}$.

\section{References}

1. Armstrong, J.: OFDM for Optical communications. Journal of Lightwave Technology 27(3) (February 1, 2009)

2. Weinstein, S., Ebert, P.: Data transmission by frequency-division multiplexing using the discrete Fourier transform. IEEE Trans. Commun. 19, 628-634 (1971)

3. Russell, M., Stüber, G.L.S.: Interchannel interference analysis of OFDM in a mobile environment. In: Proc. VTC 1995, Chicago, IL, pp. 820-824 (July 1995)

4. Sathananthan, K., Tellambura, C.: Performance analysis of an OFDM system with carrier frequency offset and phase noise. In: IEEE Vehicular Technology Conference, VTC 2001 Fall, Atlantic City, NJ, USA, October 7-11, vol. 4, pp. 2329-2332 (2001)

5. Zhao, Y., Haggman, S.-G.: Intercarrier interference self-cancellation scheme for OFDM mobile communication systems. IEEE Trans. Commun. 49, 1185-1191 (2001)

6. Ryu, H., Li, Y., Park, J.: An Improved ICI Reduction Method in OFDM Communication System. IEEE Transactions on Broadcasting 51(3) (September 2005) 